\title{
Reliability of thick Al wire: A study of the effects of wire bonding parameters on thermal cycling degradation rate using non-destructive methods
}

\author{
E. Arjmand*, P. Agyakwa, C.M. Johnson
}

Department of Electronic and Electrical Engineering, University of Nottingham, Nottingham NG7 2RD, UK

*Corresponding author: eexea8@ nottingham.ac.uk, Tel: +44 (115) 846 6890; Fax: +44 (115) 5552000

\begin{abstract}
The effect of bonding parameters on the reliability of thick $\mathrm{Al}$ wire bond is investigated. Samples were prepared with 25 different designs with 5 different bonding parameters such as time, ultrasonic power, beginforce, end-force and touch-down steps (pre-compression) with 5 levels. The bond signals of ultrasonic generator were collected during bonding in order to obtain prior quality information of bonded wires. 3D $\mathrm{x}$-ray tomography was then used to evaluate bond quality during passive thermal cycling between $-55^{\circ} \mathrm{C}$ and $125^{\circ} \mathrm{C}$. Tomography datasets were obtained from the as-bonded condition and during cycling. The results clearly show ultrasonic power, appropriate levels of begin-force and touch-down steps are all important for achieving a well attached and reliable bond. Analysis of the virtual cross-sections indicates a good correlation between the bond signal (i.e. the initial bond quality) and wire bond damage/ degradation rate. An improved understanding of the wire bonding process was achieved by observing the effect of the complex interaction of bonding parameters on the ultrasonic generator signals and degradation rate under thermal cycling.
\end{abstract}

Keywords: wire bonding process parameters, non-destructive methodology, reliability, x-ray tomography, passive thermal cycling, power electronic modules

\section{Introduction}

Power electronic modules, based on a range of semiconductor technologies are widely used to control and convert electrical power. A power electronic module has an inhomogeneous structure made up of several layers of materials such as a copper base plate, ceramic substrate, conductors, semiconductors, polymer, metal bonds and composite materials (see Fig. 1).

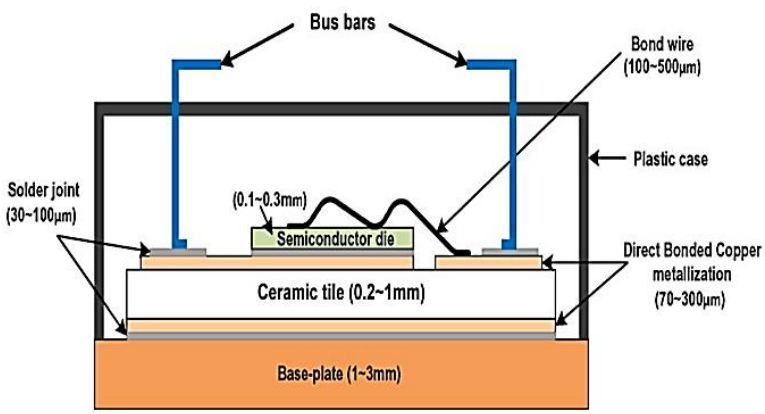

Fig. 1: Cross section view of power module [1]
These above materials are assembled together using different techniques, such as wire bonding, soldering, direct bonding copper (DBC) and pressure contact interconnect technique in the packaging manufacturing process. During their operation, power electronic modules may dissipate large amounts of heat, and maybe in service in harsh environments. Therefore, the temperature of the power electronic modules fluctuates over time. Since there are several different materials and joining technologies involved in the power electronic module, the differences in the thermal expansion coefficients cause the materials to expand and contract at different rates [2]. The stress and strain which build up as a result lead to degradation and failure of the interconnections and finally the modules. So, it is important for power electronic packaging industries to address the resulting reliability issues at the design stage and on the manufacturing line [3].

Wire bond lift-off and bond heel cracking are probably considered the most important factors in 
power electronic module reliability. Consequently, the effective detection of imperfections and reasonable bond quality evaluation has been a concern for many years; wire bond reliability strongly depends on bonding parameters such as time, ultrasonic power, force and bonding temperature. Over the last few years, there has been some work on the effect of bonding parameters on reliability of heavy wire bonds [4, 5]; however, these have not been investigated in sufficient detail considering the complex interactions between important process parameters, and more importantly with a non-destructive methodology [6].

In this work we investigate bond reliability for 5 important bond parameters with 5 levels using two analytical techniques and passive thermal cycling for evaluating the wire bonds' lifetime.

The analytical techniques included: monitoring bond quality using signals obtained from the ultrasonic bonding generator; a visual and semiquantitative characterization of virtual bond cross sections in terms of area and shape of the wire bonds and a tweezer pull test method to check bond lift-off rate.

This work allows process engineers to choose the most appropriate bonding parameter settings in an effective way by using the combination of two non-destructive methodologies.

\section{Experiment}

2.1. Evaluation of bond quality using electrical signal

In the wire bonding process, shear and pull tests are the main tests usually used to evaluate wire bond strength and quality. Both tests are not able to judge bonds strength efficiently [6]; furthermore, they are destructive and the sacrificed bonds cannot be evaluated over the entire duration of the experiment.

Recent work has investigated the use of signals obtained from the ultrasonic generator of a wire bonding machine as an indicator of bonding quality. For the first time, J. L. Landes [7] evaluated the amount of energy passing through the package. Chan et al. [8] measured the current envelope of ultrasonic signals. Or et al. [9] measured the mechanical vibrations of the transducer due to piezoelectric effect. Gibson et al. and Pufall [10, 11] also worked with a similar approach. In our own previous work, [12] we used the ultrasonic current frequency spectrum to identify wire bond quality in different simulated conditions of 'good', 'weak' and 'non- stick' bonds. Most relevantly, Wuwei et al. [13] presented a technique for on-line detection of wire bonding quality by a new feature extraction method.

In more detail, during the formation of a bond, the impedance and resonance frequency of the system are affected by the condition of the wire bond interface. Any changes in boundary condition at the tip, such as changes in the mechanical properties of the material at the interface lead to change in the electrical signals of the ultrasonic generator. The signal can reflect directly this change by the electromechanical coupling effect [13]. Therefore, electrical signals from ultrasonic generator can be used as an indicator of bonding quality.

The bond signals of the bonds on silicon dies (see Fig. 2) were collected at a sampling rate of 12.5 MHz's. A typical signature of a bonding signal and its corresponding envelope is shown in Fig. 3.

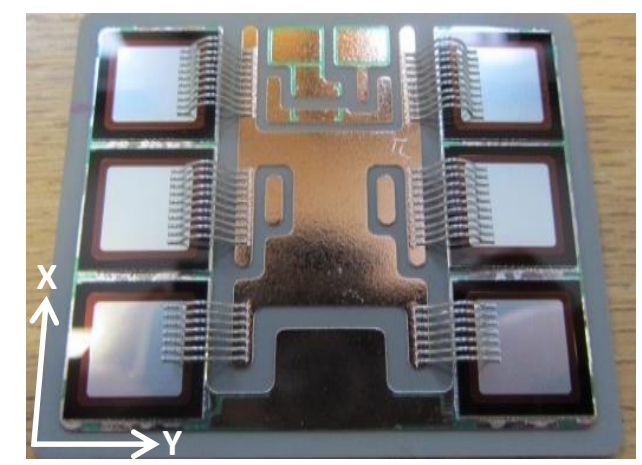

Fig. 2: Aluminium wires bonded onto silicon dies

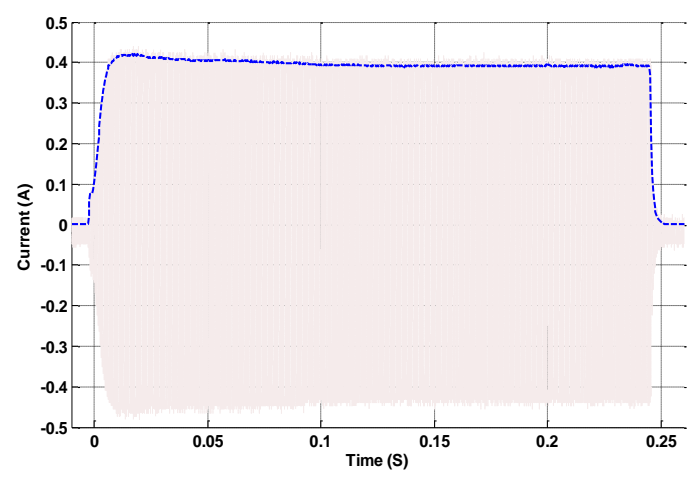

Fig. 3: A typical signature of bond signal and its corresponding envelope

\section{2. Experimental Procedure}

The test equipment in this work consists of a semi-automatic wedge bonder manufactured by F\&K 
Delvotec that operates at a signal frequency of about $58 \mathrm{kHz}$. The ultrasonic generator has a phase locked loop (PLL) controller in order to adjust the frequency output to the resonant frequency of mechanical system including the transducer, tool and sample. The generator runs in constant-voltage mode, so, the current signal varies according to the mechanical impedance presented to the transducer and it is this signal that is therefore acquired. The wire bonding signal detecting principle consists of a measuring circuit between the ultrasonic generator and transducer, an oscilloscope as a data acquisition unit and a computer for data analysis. A Versa-XRM 500 machine supplied by Carl Zeiss X-ray Microscopy was used for X-ray tomography. 25 designs were created to investigate the impact of five major bonding parameters on bond quality: namely time, ultrasonic (US) power, begin force, end force and touch-down steps (pre-compression). The designs are based on five factors with five levels. The experiment was repeated six times, making a total of 150 bonds. The bond wires were $99.999 \%$ pure $(5 \mathrm{~N})$ aluminium wires, $375 \mu \mathrm{m}$ in diameter, and were ultrasonically bonded at room temperature onto silicon dies with a 5- $\mu$ m-thick aluminium top metallization (see Fig. 2). The experimental design is shown in Table 1.

Table 1: Bonding parameters designs for reliability test

\begin{tabular}{|c|c|c|c|c|c|}
\hline $\begin{array}{c}\text { Design } \\
\text { ID }\end{array}$ & Time & $\begin{array}{c}\text { US- } \\
\text { Power }\end{array}$ & $\begin{array}{c}\text { Begin- } \\
\text { Force }\end{array}$ & $\begin{array}{c}\text { End- } \\
\text { Force }\end{array}$ & TD-Step \\
\hline $\mathbf{1}$ & 100 & 115 & 200 & 200 & 70 \\
\hline $\mathbf{2}$ & 100 & 125 & 400 & 400 & 80 \\
\hline $\mathbf{3}$ & 100 & 135 & 600 & 600 & 90 \\
\hline $\mathbf{4}$ & 100 & 145 & 800 & 800 & 100 \\
\hline $\mathbf{5}$ & 100 & 155 & 1000 & 1000 & 110 \\
\hline $\mathbf{6}$ & 135 & 115 & 400 & 600 & 100 \\
\hline $\mathbf{7}$ & 135 & 125 & 600 & 800 & 110 \\
\hline $\mathbf{8}$ & 135 & 135 & 800 & 1000 & 70 \\
\hline $\mathbf{9}$ & 135 & 145 & 1000 & 200 & 80 \\
\hline $\mathbf{1 0}$ & 135 & 155 & 200 & 400 & 90 \\
\hline $\mathbf{1 1}$ & 170 & 115 & 600 & 1000 & 80 \\
\hline $\mathbf{1 2}$ & 170 & 125 & 800 & 200 & 90 \\
\hline $\mathbf{1 3}$ & 170 & 135 & 1000 & 400 & 100 \\
\hline $\mathbf{1 4}$ & 170 & 145 & 200 & 600 & 110 \\
\hline $\mathbf{1 5}$ & 170 & 155 & 400 & 800 & 70 \\
\hline $\mathbf{1 6}$ & 215 & 115 & 800 & 400 & 110 \\
\hline $\mathbf{1 7}$ & 215 & 125 & 1000 & 600 & 70 \\
\hline $\mathbf{1 8}$ & 215 & 135 & 200 & 800 & 80 \\
\hline $\mathbf{1 9}$ & 215 & 145 & 400 & 1000 & 90 \\
\hline $\mathbf{2 0}$ & 215 & 155 & 600 & 200 & 100 \\
\hline $\mathbf{2 1}$ & 250 & 115 & 1000 & 800 & 90 \\
\hline $\mathbf{2 2}$ & 250 & 125 & 200 & 1000 & 100 \\
\hline $\mathbf{2 3}$ & 250 & 135 & 400 & 200 & 110 \\
\hline $\mathbf{2 4}$ & 250 & 145 & 600 & 400 & 70 \\
\hline $\mathbf{2 5}$ & 250 & 155 & 800 & 600 & 80 \\
\hline & & & & & \\
\hline
\end{tabular}

The bonds were subjected to passive thermal cycling from -55 to $+125^{\circ} \mathrm{C}$. From the 150 bonds, 25 were randomly selected for $\mathrm{x}$-ray tomography and imaged. The remaining 125 bonds were selected for tweezer test after every 100 cycles in order to record any lift-offs or failures.

\section{Results and Discussions}

3.1. Relation of different designs and bonds attached area

The 25 bonds that were selected for $\mathrm{x}$-ray tomography were imaged in the as-bonded condition (zero cycles), after 700 cycles. From a virtual cross section of bond interface in the X-Y plane (see Fig. 4) the attached area in the as-bonded condition was measured using ImageJ software. Results are given in Fig. 5.

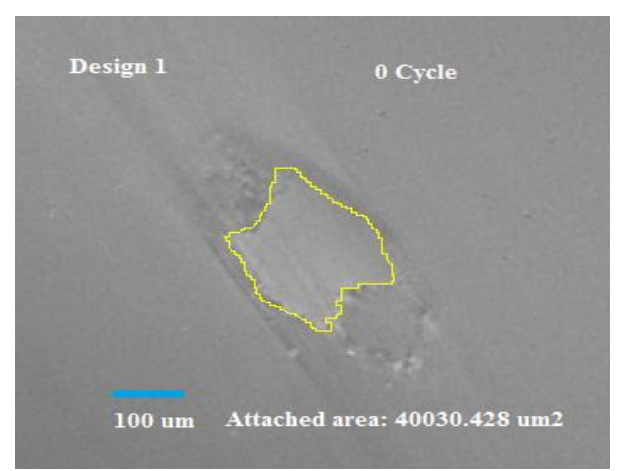

Fig. 4: X-ray tomography image showing the bonded area of Design 1

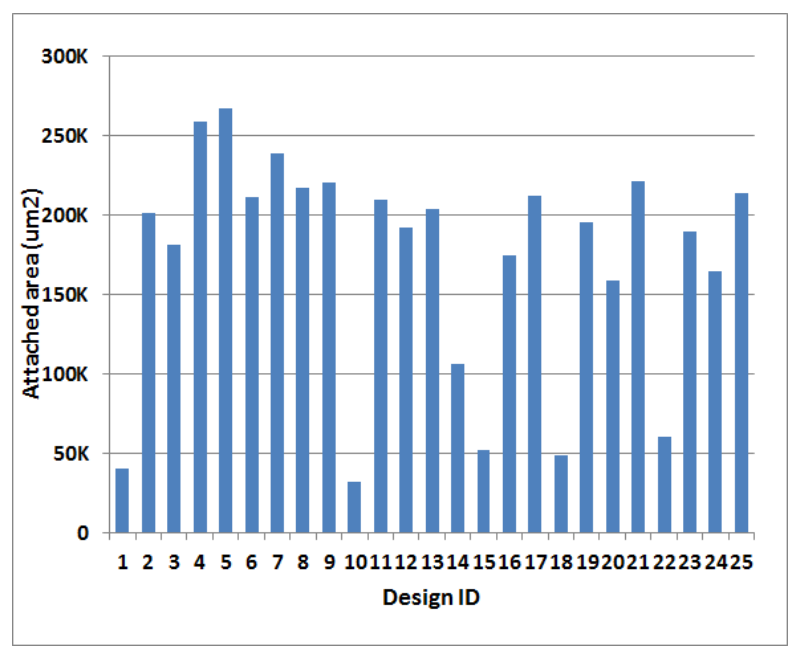

Fig. 5: Bond attached area for different parameter designs 
From the tomography images in the as-bonded condition, designs $10,1,18$ and 15 have the least attached area, and designs 5, 4, 7 and 21 have the largest attached area.

All the bonded wires were then subjected to thermal cycling from -55 to $+125{ }^{\circ} \mathrm{C}$. Tweezer pull test results of 125 wires are given in Fig. 6 .

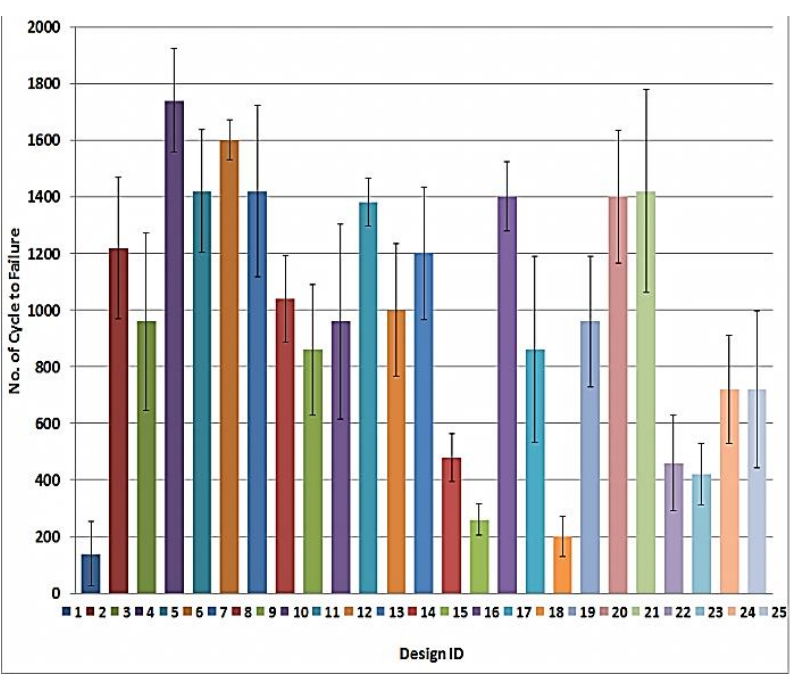

Fig. 6: Result of bonds lift-off rate after thermal cycling of different parameter designs

Result of bond lift-off rate indicates that the bonds with least attached area are less reliable, such as designs 1, 18 and 15, and bonds with largest attached area are more reliable. From both x-ray tomography images and bond lift-off rate following results are found:

1) Insufficient begin force and touch-down steps (pre-compression) result in poor attached area and ultimately less reliable bonds, such as design 1, 18, 22, and 15 (see Fig. 7). As can be seen in Fig. 7, in the as-bonded condition the bonds attached from the middle with nonuniform shape and pre-cracks appear from both corners of the bonds. After 700 cycles the cracks started to grow from both corners and microvoids started to join together, and as shown in the $\mathrm{X}-\mathrm{Z}$ plane image of design 1 , the bond had almost lifted off.
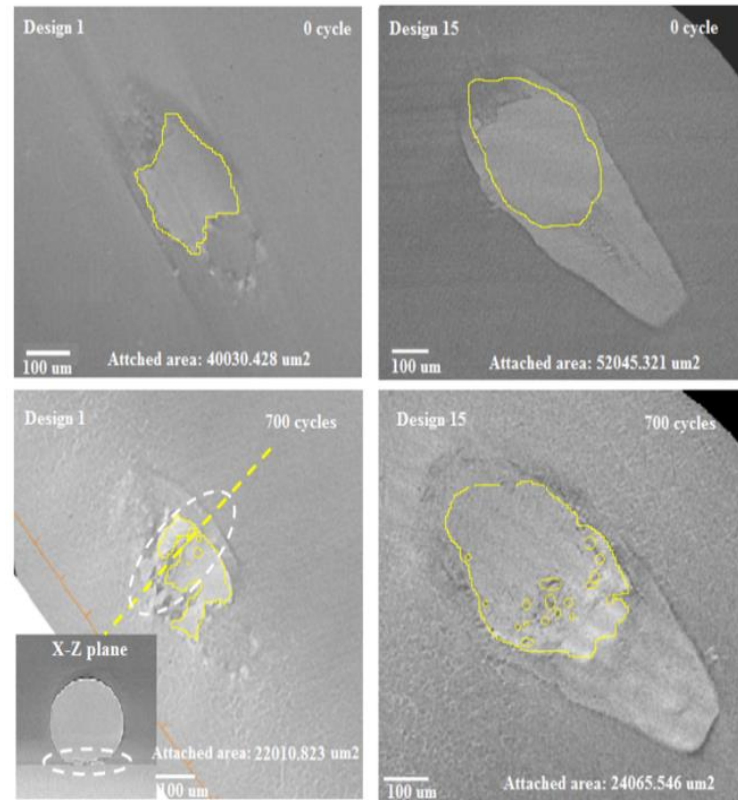

Fig. 7: X-ray tomography images of design 1 and 15 in X-Y plane in as-bonded condition and after 700 cycles

2) Sufficient US power and force create uniform attached area and more reliable bonds such as designs 4, 6, 21, 7 and 5 (see Fig. 8 and 6).
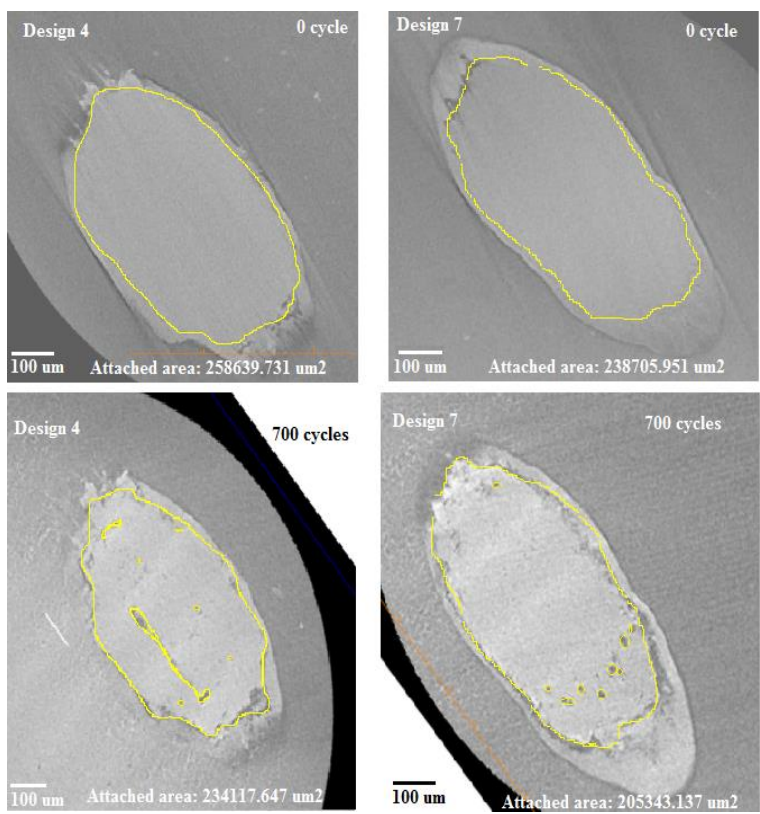

Fig. 8: X-ray tomography images of design 4 and 7 in the $\mathrm{X}-\mathrm{Y}$ plane in as-bonded condition and after 700 cycles 
3) Results of designs 21 and 4 show that there is an inverse relation between US power and time. It can be said that you can get well attached and reliable bonds with high power in lowest time and or less power with longer time. Thus the total energy is an important factor, rather than either power or time.

Altogether, the 3D x-ray images allow the process engineer to observe bond morphology and damage growth rate throughout the test period. However, it is expensive and time consuming and cannot be justified for all bonded wires in a production environment.

3.2. Relation of different designs and bond electrical signal

From the above experiment, with its complexity of designs, it was seen that there are many poor bonding parameter settings that make the bonds less reliable while there are few parameter combinations that make the bonds well attached and hence more reliable. It is therefore of interest to investigate whether a single parameter, namely the current signature of ultrasonic generator, can be used as an indicator of bond quality. For instance, as it can be seen in Fig. 9 that all current signals rise sharply for about 2 milliseconds at beginning of bonding before diverging. Different settings and combinations of parameters lead to differences in the rising characteristics and uniformity of the waveforms, the time they take to reach a steady state and the maximum current (see Fig. 9). These features allow potential correlations between the bond quality and current signal to be investigated.

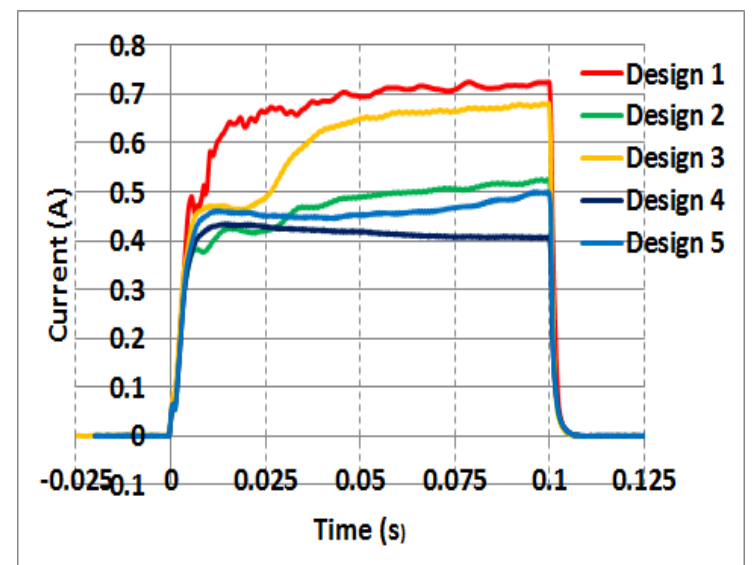

Fig. 9: Current envelope of bonding signals for designs 1 to 5 .
From the result of bonding parameters designs that are shown in figures 5 and 6 , the following remarks are found:

1) For the bonding parameter designs that create less attached bonded area and ultimately shorter lifetimes, current signals reach a steady state at a higher value compared to the designs that have more attached area and longer lifetime (see Fig. 10). Furthermore, the result obtained from signal analysis show the more reliable designs such as design 4 have more uniform signal shape compared to less reliable designs such as design 1 .

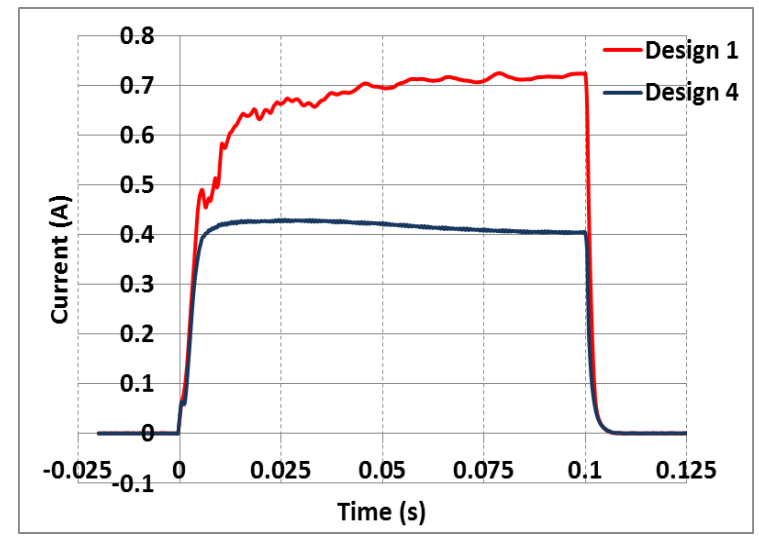

Fig. 10: Current envelope of bonding signals for designs 1 and 4 (weakest design compare strongest design ).

2) It can be said that the bonding parameter designs such as $4,6,21,7$ and 5 received a more constant level of power compared to the other designs (See Fig. 11), indicating consistent mechanical conditions at the bond foot. The $\mathrm{x}$ ray tomography images and lift-off rates confirm that these are more reliable designs. Average lifetimes of the above designs were 1740, 1600, 1420, 1420 and 1420, respectively. 


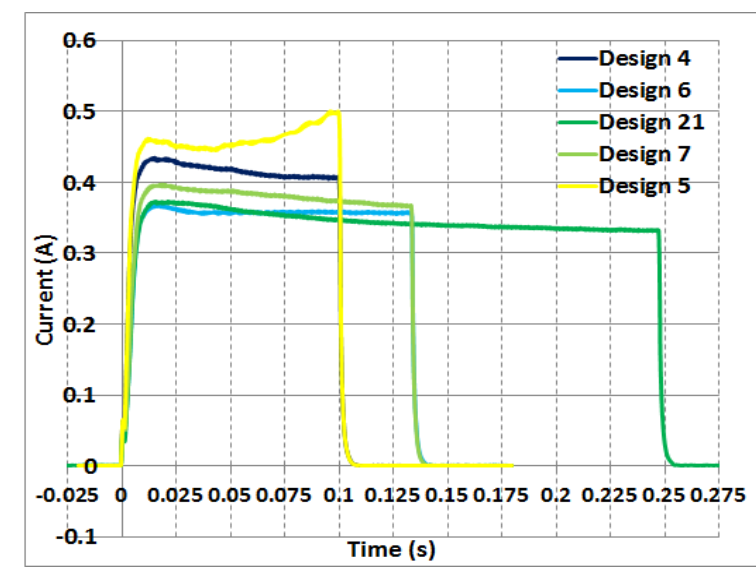

Fig. 11: Current envelope of bonding signals for the most reliable designs.

3) A number of designs such as 1, 18, 15, 23 and 22 have unstable signals during bonding. Such signal characteristics indicate inconsistent transfer of energy at the bond interface, probably resulting from changing mechanical conditions. This could be because of inappropriate levels of begin-force and touch down steps (pre-compression). The lift-off rates during thermal cycling also confirm that the above designs are the weakest designs (see Fig. 12). Average lifetimes of mentioned designs are 140, 200, 260, 420 and 460 cycles, respectively (see Fig. 6).

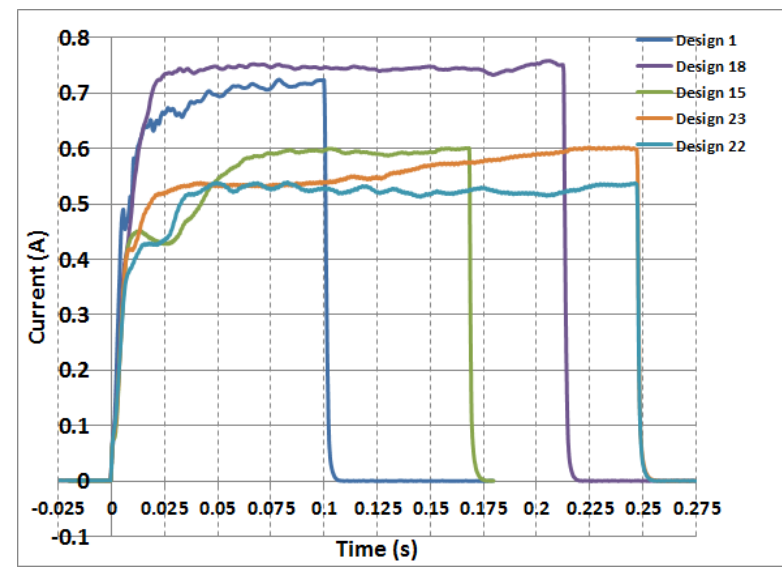

Fig. 12: Current envelope of bonding signals for the less reliable designs

4) Interestingly, the correlation between beginforce and end-force with electrical signal can be seen clearly when begin-force value is lower than end-force, and vice versa (see Fig. 13). For instance, in design 14 the begin-force was set to a low value of $200 \mathrm{cN}$, the signal rose to a high level as for other weak designs, then slowly fell to a lower value and finally, after the higher end-force was applied, levelled off. Thus the changing mechanical conditions at the bond foot are directly reflected in the current envelope. Results of thermal cycling and tomography confirm the importance of proper begin-force on reliability of wire bonds. As can be seen in Fig. 6 , the bonds in designs 14 and 12 survived an average of 480 and 1000 cycles, respectively.

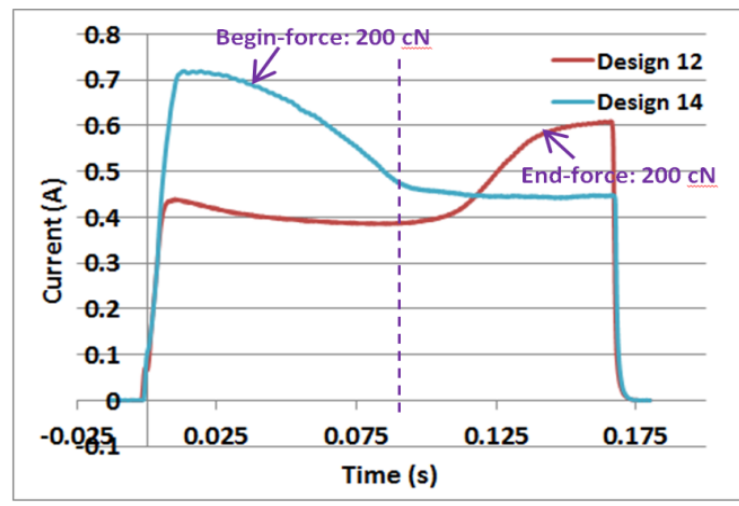

Fig. 13: Current envelope of bonding signals for designs 14 and 12 .

5) Signal analysis also allows us to observe the repeatability of bonding of the different designs. For instance, as it can be seen in Fig. 14, design 17 shows different signals for each trial, which indicates the design is not repeatable. Entirely different lifetimes resulted from this cohort (see Fig. 6). It is likely that this variability results from the low number of touch-down steps (precompression) used in this design.

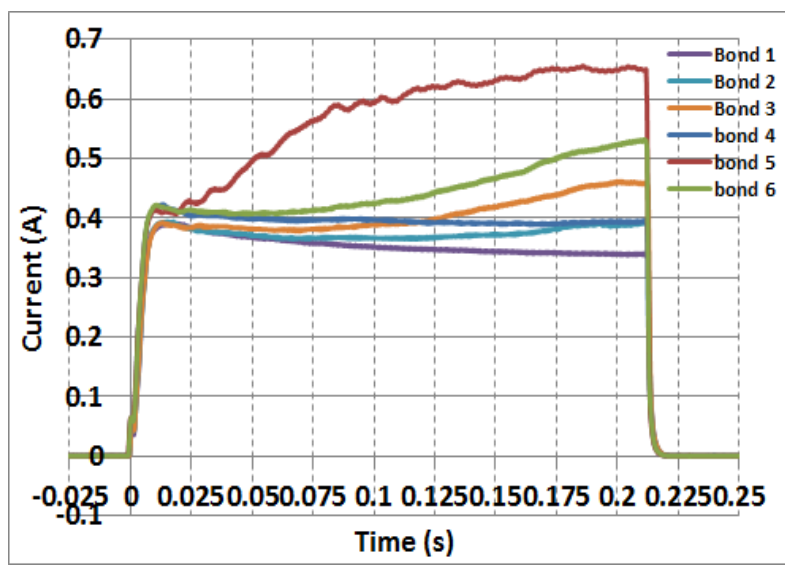

Fig. 14: Current envelope of bonding signals for the designs 17 


\section{Summary and Conclusions}

In this paper, the effects of bonding parameters on the reliability of wire bonds in power electronic modules have been investigated. The most obvious finding to emerge from this work are: 1) different setting and combinations of parameters lead to different in rising characteristics and uniformity of waveforms, 2) a constant level of power indicates consistent mechanical conditions at the bond foot resulting in large attached area and ultimately longer lifetime, 3) inconsistent energy transfer observed, for example when inappropriate levels of begin-force and/or touch-down steps (pre-compression) is used, result in poor bond quality. Taken together, these results suggest that the ultrasonic power, appropriate levels of begin-force and touch-down steps are all important for achieving a well attached and reliable bond. Overall, we have demonstrated that bond signal analysis can facilitate the non-destructive evaluation of the effect of the bonding process parameters on bond quality. It is fast, cheap and can be used on-line in the manufacturing process. Furthermore, X-ray tomography images of bonds indicate a strong correlation between the inferences of bond quality made from the bond signals and wire bond lifetime. In future work, it would be interesting to see whether different designs lead to the creation of different defects during thermal cycling. Further, the reduction of attached area will be measured, to obtain the rate of degradation for the different designs. Overall, both bond signal analysis and Xray tomography are suitable non-destructive methodologies for investigating the complex effects of process parameters on the reliability of wire bonds.

\section{Acknowledgements}

The authors gratefully acknowledge the support of the UK Engineering and Science Research Council (EPSRC) through grant EP/I013636/1 (HubNet). The authors are also grateful to Dynex Semiconductors Ltd. for providing the dies and DBC substrate tiles for this work.

\section{References}

[1] Y. Li, P. A. Agyakwa, and C. M. Johnson, "Physics-of-Failure Lifetime Prediction Models for Wire Bond Interconnects in Power Electronic Modules," Device and Materials Reliability, IEEE Transactions on, vol. 13, pp. 9-17, 2013.
[2] L. Hua and C. Bailey, "Lifetime prediction of an IGBT power electronics module under cyclic temperature loading conditions," in Electronic Packaging Technology \& High Density Packaging, 2009. ICEPT-HDP '09. International Conference on, 2009, pp. 274-279.

[3] C. Bailey, H. Lu, C. Yin, and S. Ridout, "Predictive Reliability, Prognostics and Risk Assessment for Power Modules," Integrated Power Systems (CIPS), 2008 5th International Conference on, pp. 1-7, 2008.

[4] L. Wei-Sun, M. Corfield, L. Hua, S. Hogg, T. Tilford, and C. M. Johnson, "Wire Bond Reliability for Power Electronic Modules Effect of Bonding Temperature," in Thermal, Mechanical and Multi-Physics Simulation Experiments in Microelectronics and MicroSystems, 2007. EuroSime 2007. International Conference on, 2007, pp. 1-6.

[5] J. Goehre, U. Geissler, M. Schneider-Ramelow, and K. Lang, "Influence of Bonding Parameters on the Reliability of Heavy Wire Bonds on Power Semiconductors," in Integrated Power Electronics Systems (CIPS), 2012 7th International Conference on, 2012, pp. 1-6.

[6] L. Y. Pearl A Agyakwa*, Martin R Corfield \& C Mark Johnson, "A nondestructive study of crack development during thermal cycling of Al wire bonds using x-ray computed tomography," presented at the CIPS 2014 Germany, 2014.

[7] J. L. Landes, "Ultrasonic bond energy monitor," U.S. Classification Patent, 1982.

[8] L. K. Chan, "Apparatus and method for automatic evaluation of a bond created by an ultrasonic transducer," US Patent, 1982.

[9] S. W. Or, H. L. W. Chan, V. C. Lo, and C. W. Yuen, "Ultrasonic wire-bond quality monitoring using piezoelectric sensor," Sensors and Actuators A: Physical, vol. 65, pp. 69-75, 1998.

[10] W. J. G. O. E. Gibson, L. D. Burkholder and B. K. Benton, "Bond Signature Analyzer," US Patent, 1989.

[11] R. Pufall, "Automatic process control of wire bonding," in Electronic Components and Technology Conference, 1993. Proceedings., $43 r d, 1993$, pp. $159-162$.

[12] E. Arjmand, P. Agyakwa, and C. M. Johnson, "Methodology for identifying wire bond process quality variation using ultrasonic current frequency spectrum," in Power Electronics and Applications (EPE), 2013 15th European Conference on, 2013, pp. 1-8.

[13] W. Feng, Q. Meng, Y. Xie, and H. Fan, "Wire bonding quality monitoring via refining process of electrical signal from ultrasonic generator," Mechanical Systems and Signal Processing, vol. 25, pp. 884-900, 2011. 